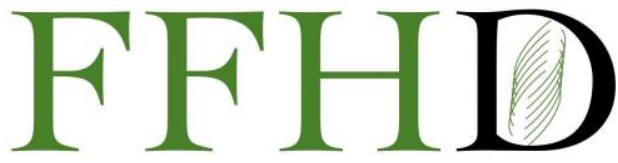

Functional Foods in Health and Disease

\title{
Black soybean seed coat polyphenols promote nitric oxide production in the aorta through the Akt/eNOS pathway
}

\author{
Chiaki Domae, Hitoshi Ashida, Yoko Yamashita*
}

Department of Agrobioscience, Graduate School of Agricultural Science, Kobe University, Nada-ku, Kobe, Hyogo 6578501, Japan

*Corresponding author: Yoko Yamashita, Department of Agrobioscience, Graduate School of Agricultural Science, Kobe University, Nada-ku, Kobe, Hyogo 657-8501, Japan

Submission Date: June 12 ${ }^{\text {th }}, 2020$; Acceptance Date: July $24^{\text {th }}, 2020$; Publication Date: August $13^{\text {th }}, 2020$

Please cite this article as: Domae C., Ashida H., and Yamashita Y. Black Soybean seed coat polyphenols promote nitric oxide production in the aerta through the Akt/eNOS pathway. Functional Foods in Health and Disease 2020; 10(8): 330-343. DOI: https://doi.org/10.31989/ffhd.v10i8.722

This article is a part of special issue of ICoFF/ISNFF 2019, Kobe, Japan. Special issue editors: Yasuhito Shirai, PhD, Professor, Graduate School of Agricultural Science, Department of Agrobioscience, Kobe University, Kobe, Japan and Hiroshi Yoshida, MD, PhD, Professor, The Jikei University School of Medicine, Tokyo, Japan

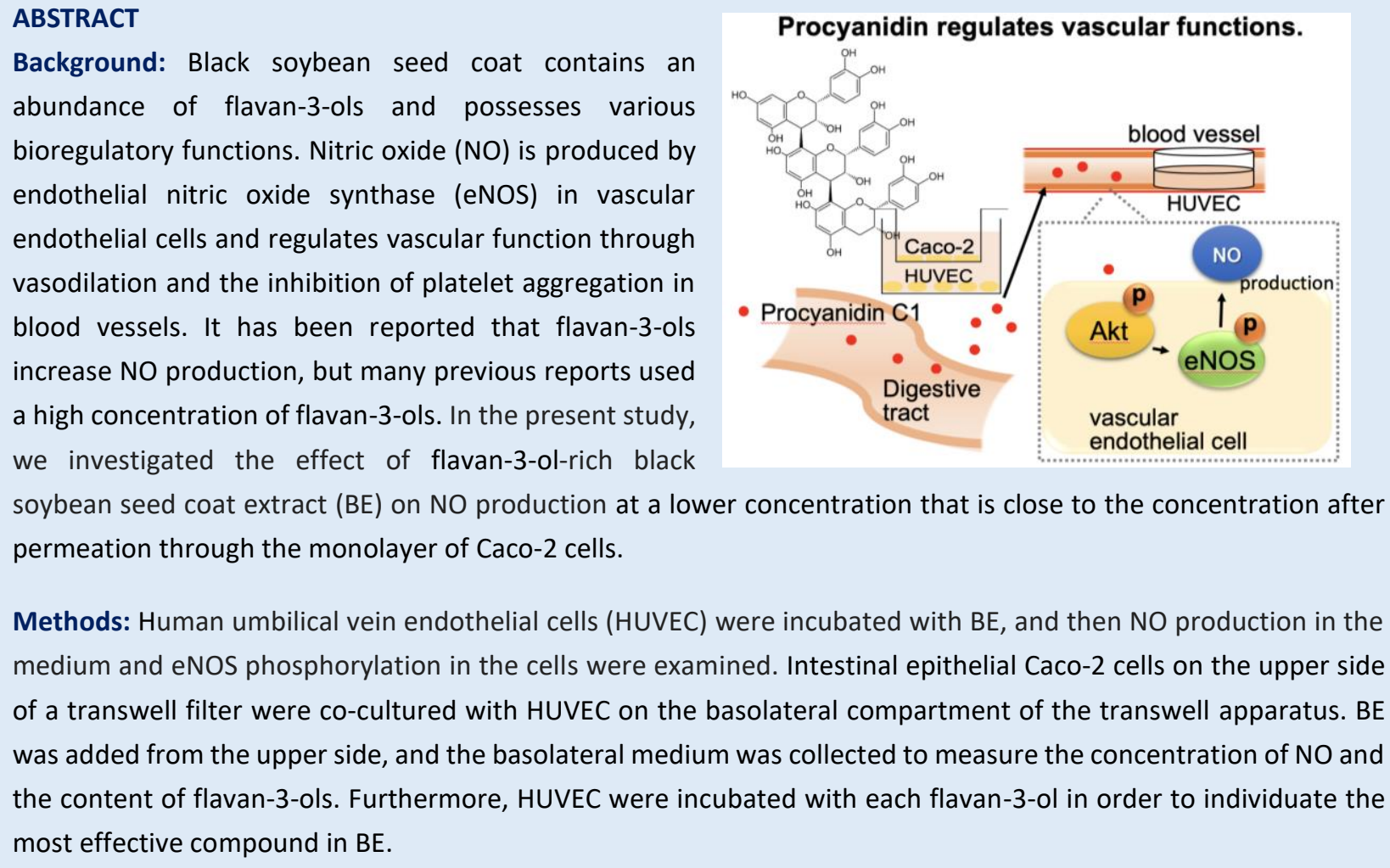

soybean seed coat extract (BE) on NO production at a lower concentration that is close to the concentration after permeation through the monolayer of Caco-2 cells.

Methods: Human umbilical vein endothelial cells (HUVEC) were incubated with BE, and then NO production in the medium and eNOS phosphorylation in the cells were examined. Intestinal epithelial Caco-2 cells on the upper side of a transwell filter were co-cultured with HUVEC on the basolateral compartment of the transwell apparatus. BE was added from the upper side, and the basolateral medium was collected to measure the concentration of NO and the content of flavan-3-ols. Furthermore, HUVEC were incubated with each flavan-3-ol in order to individuate the most effective compound in BE. 
Results: BE significantly increased NO production in the medium of HUVEC. When polyphenols in BE were removed from the basolateral medium by ethyl acetate extraction, increased NO production from HUVEC was not observed. Additionally, BE increased phosphorylation of eNOS and Akt in HUVEC. A portion of flavan-3-ols in BE had permeated through intestinal epithelial cells. Among the flavan-3-ols that had permeated, procyanidin $\mathrm{C} 1$ had the strongest effect on NO production in HUVEC at the concentration that had permeated the monolayer of Caco-2 cells. Procyanidin C1 (0.05 $\mu \mathrm{M})$ also induced phosphorylation of eNOS and Akt in HUVEC without affecting the cAMP level.

Conclusion: A portion of flavan-3-ols in BE directly promoted NO production through the Akt/eNOS pathway in vascular endothelial cells. These findings suggest that flavan-3-ols in the black soybean seed coat may contribute to improve the vascular function.

Keywords: Black soybean seed coat polyphenols; NO; eNOS; Akt; vascular endothelial cells

CFFC 2020. This is an Open Access article distributed under the terms of the Creative Commons Attribution 4.0 License (http://creativecommons.org/licenses/by/4.0)

\section{INTRODUCTION}

Vascular endothelial cells play an important role in maintaining vascular homeostasis. It is known that diabetes, obesity, and oxidative stress cause damage to vascular endothelial cells and lead to vascular endothelial dysfunction [1, 2, 3, 4]. Moreover, aging and vascular stiffness, which are associated with vascular dysfunction, are risk factors of cardiovascular diseases (CVD). It is, therefore, important to search for food factors that can prevent and ameliorate vascular dysfunction.

Vascular homeostasis is regulated mainly by the balance of paracrine factors, such as nitric oxide (NO) [5, 6]. NO is produced by inducible nitric oxide synthase (iNOS), neuronal nitric oxide synthase (nNOS), and endothelial nitric oxide synthase (eNOS). nNOS and eNOS are constitutively expressed mainly in the nerve cells and the vascular endothelial cells, respectively. Inflammatory stimuli induce iNOS to overproduce NO, which can cause oxidative stress, oncogenesis, inflammation, and more [7]. On the other hand, NO produced by eNOS in vascular endothelial cells contributes to the regulation of vascular function. eNOS activation is regulated by several molecular mechanisms, including $\mathrm{Ca} 2+/$ calmodulin binding $[5,8]$,
CAMP-dependent protein kinase, AMP-activated protein kinase (AMPK) [5], and Akt [5, 8]. Akt promotes the phosphorylation of eNOS at Ser1177 residues in response to various stimuli, such as insulin [9].

Black soybean seed coat contains an abundance of flavan-3-ols and anthocyanins, including epicatechin, procyanidin B2, procyanidin C1, cinnamtannin A2 and other polymer procyanidins, $[10,11]$. It has been reported that these flavan-3-ols in the black soybean seed coat have beneficial health effects, such as antioxidant [12], anti-obesity, and anti-diabetic activities [13]. Recently, we have reported that an intake of roasted black soybean improved vascular function by increasing NO production in humans [14]. Moreover, we have found that black soybean seed coat extract $(B E)$ increased NO production and GLP-1 secretion from the intestine of rats [15]. Although bioavailability of flavan-3-ols is poor, it has been reported that (-)-epicatechin and small amounts of oligomer procyanidins such as procyanidin B2, procyanidin $\mathrm{C} 1$ and cynnamtannin $\mathrm{A} 2$ in $\mathrm{BE}$ were absorbed from the intestinal tracts and appeared in the plasma and various tissues after the oral administration of $B E$ in mice [16]. Thus, it is expected that certain flavan-3-ols in BE may promote NO production directly 
in vascular endothelial cells after absorption from the intestinal tracts. Other reports also demonstrate that flavan-3-ols increase NO production via both the Akt/eNOS pathway and Ca2+ dependent pathway in vitro [17-19]. However, it is unclear whether $B E$ promotes NO production at a lower concentration that is close to plasma or tissue concentration after their absorption. Therefore, we investigated the effect of $B E$ and its flavan-3-ols on NO production in human umbilical vein endothelial cells (HUVEC) and its underlying mechanism at a lower concentration that is close to the concentration after permeation through a monolayer of Caco-2 cells.

\section{METHODS}

Materials: BE, a product of Fujicco Co., Ltd. (Kobe, Japan), was prepared by extraction with acidic water and ethanol according to a previous method [20], and its polyphenol composition was measured by highperformance liquid-chromatography [16]. BE consisted of $6.2 \%$ epicatechin, $39.7 \%$ procyanidin $(6.1 \%$ procyanidin $\mathrm{B} 2, \quad 3.4 \%$ procyanidin $\mathrm{C} 1$, and $0.5 \%$ cinnamtannin A2), 9.2\% cyanidin 3-glucoside and unidentified polyphenols including highly-polymerized procyanidins (degree of polymerization $\geq 5$ ). Total amount of polyphenols in BE was $67.0 \%$, which was determined by the Folin-Denis method [21].

LY294002 was purchased from FUJIFILM Wako Pure Chemical Corporation (Osaka, Japan). Antibodies against p-eNOS (Ser1177), p-Akt (Ser473), p-Akt (Thr308), Akt, and $\beta$-actin were obtained from Cell Signaling Technology (Danvers, MA, USA). An antibody against eNOS was sourced from Santa Cruz Biotechnology (Dallas, TX, USA). All other reagents used were of the highest grade available from commercial sources.
Cell culture: HUVEC was obtained from the Japanese Collection of Research Bioresources Cell Bank (Osaka, Japan) and grown in Ham's F-12K (Fujifilm Wako Pure Chemical Corporation, Osaka, Japan) supplemented with $20 \%(v / v)$ fetal bovine serum (Biowest, Nuaillé, France), $50 \mu \mathrm{g} / \mathrm{ml}$ endothelial cell growth supplement from bovine neural tissue (Sigma-Aldrich, St. Louis, MO, USA) and $100 \mu \mathrm{g} / \mathrm{ml}$ heparin sodium under a humidified atmosphere of $95 \%(\mathrm{v} / \mathrm{v})$ air and $5 \%(\mathrm{v} / \mathrm{v}) \mathrm{CO}_{2}$ at $37{ }^{\circ} \mathrm{C}$. Intestinal epithelial Caco-2 cell lineage was obtained from the American Type Culture Collection (Manassas, VA, USA) and grown in Dulbecco's modified Eagle's medium (Nissui Pharmaceutical, Tokyo, Japan) containing high glucose ( $4.5 \mathrm{~g} / \mathrm{L}$ glucose), $10 \%$ (v/v) fetal bovine serum, $4 \mathrm{mM}$ L-glutamine, $100 \mathrm{U} / \mathrm{ml}$ penicillin and $100 \mu \mathrm{g} / \mathrm{ml}$ streptomycin under a humidified atmosphere of $95 \%(\mathrm{v} / \mathrm{v})$ air and $5 \%(\mathrm{v} / \mathrm{v}) \mathrm{CO}_{2}$ at $37{ }^{\circ} \mathrm{C}$.

\section{Measurement of NO production in the cell culture} medium: As an indicator of NO production, the presence of nitrite in the cell culture medium was determined using the Griess method [22]. HUVEC was briefly cultured in a 24-well plate to reach confluence. The cells were pretreated with or without $10 \mu \mathrm{M}$ PI3K inhibitor (LY294002) for $1 \mathrm{~h}$ and then either BE, (-)epicatechin, procyanidin B2, procyanidin C1 and cynnamtannin A2 was added to each well for $1 \mathrm{~h}$. For the positive and negative controls, $100 \mathrm{nM}$ insulin and DMSO were used. The cell culture medium was collected, and aliquot of $150 \mu$ l of it was mixed with 75 $\mu \mathrm{l}$ of $1 \%$ sulfanilamide in $5 \%$ phosphoric acid, and an equal volume of $0.1 \% N$ - (1-naphthyl) ethylenediamine dihydrochloride in distilled water. The absorbances at $535 \mathrm{~nm}$ were measured using a Wallac 1420 ARVO Sx microplate reader (Perkin Elmer Inc., Waltham, MA, USA). A calibration curve was prepared using a 0-100 $\mu \mathrm{M}$ sodium nitrite solution as a standard. 
Western blotting analysis: HUVEC was cultured on a 35-mm dish to reach confluence. The cells were treated with either BE, (-)-epicatechin, procyanidin B2, procyanidin $\mathrm{C} 1$ and cynnamtannin $\mathrm{A} 2$ at the indicated concentrations in the figures below. Insulin at $100 \mathrm{nM}$ and DMSO were used as the positive and negative controls, respectively. The cells were washed with phosphate-buffered saline (PBS), lysed with radioimmunoprecipitation assay buffer $[10 \mathrm{mM}$ Tris- $\mathrm{HCl}$ pH 8.0, 1\% Nonidet P-40, $150 \mathrm{mM} \mathrm{NaCl}, 0.5 \%$ sodium deoxycholate, and $0.1 \%$ sodium dodecyl sulfate (SDS)] containing $0.5 \mathrm{mM}$ dithiothreitol protease inhibitors (1 $\mathrm{mM}$ phenylmethylsulfonyl fluoride, $5 \mu \mathrm{g} / \mathrm{ml}$ leupeptin, and $5 \mu \mathrm{g} / \mathrm{ml}$ aprotinin) and phosphatase inhibitors (10 $\mathrm{mM} \mathrm{NaF}$ and $1 \mathrm{mM} \mathrm{Na}_{3} \mathrm{VO}_{4}$ ), and homogenized using a hand-held microtube homogenizer. Homogenate was left on ice for $1 \mathrm{~h}$ and mixed, then centrifuged at 12,000 $\times g$ for $20 \mathrm{~min}$ at $4^{\circ} \mathrm{C}$. Obtained supernatant was used as cell lysate. Detection of each target protein was performed by western blotting. After SDSpolyacrylamide gel electrophoresis, the separated proteins in the gel were transferred onto a polyvinylidene fluoride membrane. The membrane was incubated with Blocking One (Nacalai Tesque, Kyoto, Japan) for $1 \mathrm{~h}$ at room temperature. Afterwards, the membrane was further incubated with the primary antibody overnight at $4^{\circ} \mathrm{C}$, followed by incubation with the corresponding horseradish peroxidase-conjugated secondary antibody for $1 \mathrm{~h}$ at room temperature. Primary and secondary antibodies were diluted in the blocking solution (Blocking One: TBST $=1: 19$ ) at ratio of 1:5,000 and 1:50,000, respectively. The protein bands were visualized using Immuno Star ${ }^{\circledR}$ LD (FUJIFILM Wako Pure Chemical Corporation) and detected by LightCapture II (ATTO, Tokyo, Japan). The density of a specific band was quantified using ImageJ software (National Institutes of Health, Bethesda, MD, USA).

\section{A co-culture system of Caco-2cells and HUVEC: Caco-2}

cells were cultured on a 24-well culture insert of a transwell apparatus (BD Biosciences, Erembodegem, Belgium) at a density of $6 \times 10^{4}$ cells per insert, and the cells were used from the $15^{\text {th }}$ day to the $19^{\text {th }}$ day of the experiment. The transepithelial electrical resistance (TER) value of the monolayers was measured using a Millicell-ERS instrument (Millipore Corp., Bedford, MA, USA). After confirmation of differentiation by the TER value (higher than $600 \Omega \mathrm{cm}^{2}$ ), the Caco-2 cells were cocultured with HUVEC on the basolateral side of the transwell apparatus. BE at concentrations of $0.1,1$ or 10 $\mathrm{mg} / \mathrm{ml}$ was added to the apical side and incubated for $24 \mathrm{~h}$, and the cell culture medium from the basolateral side was collected to measure nitrite concentration and composition of flavan-3-ols.

\section{Influence of secretory factor from Caco-2 cell for the} NO production: $\mathrm{BE}$ at $10 \mathrm{mg} / \mathrm{ml}$ was added to Caco-2 cells on the apical side of transwell apparatus for $24 \mathrm{~h}$. Then, the medium from the basolateral side was collected. The polyphenols in the permeated medium were removed by extraction with ethyl acetate. After removing the ethyl acetate layer, the bottom layer was collected and evaporated to dryness using the centrifugal separator. The dried residue was dissolved in water, and the obtained solution was used to treat HUVEC for $1 \mathrm{hr}$ and subjected the Griess method to measure the NO concentration.

HPLC analysis: To determine the levels of flavan-3-ols that permeated through Caco-2 cells, the collected medium from the basolateral side of the transwell apparatus was analyzed by HPLC with or without deconjugation reaction using glucuronidase and sulfatase [16]. HPLC was performed according to a previous method [23] using a SHIMADZU LabSolutions 
system (SHIMADZU, Kyoto, Japan) with SPD-M20A diode array detector, equipped with a column [Cadenza CL-C18 column (\$ $250 \mathrm{~mm} \times 4.6 \mathrm{~mm}, 3 \mu \mathrm{m}$, Imtakt, Kyoto, Japan)]. HPLC separation was done with a gradient system using $0.1 \%$ formic acid solution as mobile phase $A$ and acetonitrile as mobile phase $B$. Separation of procyanidins was achieved using a linear gradient from 5-15\% B over 0-45 minutes; $15-80 \%$ B over $45-50$ minutes; $80 \%$ B over 50-53 minutes; and 5\% B over 53-70 minutes. The flow rate was set at 0.7 $\mathrm{ml} / \mathrm{min}[23]$.

\section{Measurement of intracellular $\mathrm{Ca}^{2+}$ levels: HUVEC was} cultured in a 12-well plate with coverslip up to reach confluence. The cells were washed three times with PBS, and incubated in a recording medium (20 mM HEPES, $115 \mathrm{mM} \mathrm{NaCl}, 5.4 \mathrm{mM} \mathrm{KCl}, 13.8 \mathrm{mM}$ glucose, $0.8 \mathrm{mM}$ $\mathrm{MgCl}_{2}$ and $1.8 \mathrm{mM} \mathrm{CaCl}_{2}$ ) containing $5 \mu \mathrm{M}$ Fura2-AM (Dojindo, Kumamoto, Japan), 0.04\% Pluronic F-127 and $1.25 \mathrm{mM}$ probenecid for $1 \mathrm{~h}$ at $37^{\circ} \mathrm{C}$. The medium was removed and the cells were washed tree times with PBS. Then, the PBS was removed and replaced with the recording medium containing $1.25 \mathrm{mM}$ probenecid. After treatment with 0.05 or $50 \mu \mathrm{M}$ procyanidin $\mathrm{C} 1$ or A23187 (Calcium lonophore) as a positive control for 10 min, the medium was removed and the cells were washed three times with PBS. The cells were immobilized with $4 \%$ paraformaldehyde solution for 20 min and washed three times with PBS. Coverslips were mounted on a microscope slide by a mounting solution (glycerol containing 1\% 1,4-diazabicyclo[2,2,2]-octane and $20 \mathrm{mM}$ Tris- $\mathrm{HCl}, \mathrm{pH}$ 7.5). The fluorescence of Fura2AM was measured with an excitation wavelength at $340 / 380 \mathrm{~nm}$ and emission one at $510 \mathrm{~nm}$ using an FSX100 microscope (Olympus, Tokyo, Japan).
Statistical analysis: Data are represented as the mean \pm SE. The statistical significance of experimental observations was determined using Dunnett's multiple comparison test (Figures 1, 2, 3, and 4) and Tukey's HSD test (Figure 5). The level of significance was set at $p<$ 0.05 .

\section{RESULTS}

Effects of BE on NO production and eNOS phosphorylation in HUVEC: In order to determine whether BE promotes NO production in HUVEC, the cells were treated with BE $(0.01-100 \mu \mathrm{g} / \mathrm{ml})$ for $1 \mathrm{~h}$, after which nitrite concentration in the culture medium was used to estimate the NO production. As shown in Figure $1 \mathrm{~A}, 1-100 \mu \mathrm{g} / \mathrm{ml} \mathrm{BE}$ significantly increased NO production from HUVEC. To investigate the upstream factors of NO production, HUVEC was treated with BE (0.01-100 $\mathrm{\mu g} / \mathrm{ml})$ for $30 \mathrm{~min}$ and western blotting analysis was performed. BE $1-100 \mu \mathrm{g} / \mathrm{ml}$ significantly increased phosphorylation of Akt at Thr308 and eNOS in HUVEC (Figure 1B and C). Phosphorylation of Akt at Ser473 was also significantly increased only by BE at 100 $\mu \mathrm{g} / \mathrm{ml}$. These results suggested that BE could increase NO production via the Akt/eNOS pathway in HUVEC.

Effect of permeated flavan-3-ols in BE on NO production in a co-culture system consisting of Caco-2 cells and HUVEC: It is known that the polyphenols in BE are absorbed in small, but statistically significant amounts [16]. To take account of their low bioavailability, the effect of BE on NO production was measured in a co-culture system consisting of Caco-2 cells and HUVEC. BE $(0.1-10 \mathrm{mg} / \mathrm{ml})$ was added to the apical side of the transwell apparatus and incubated for $24 \mathrm{~h}$, and then NO production in the cell culture 
A

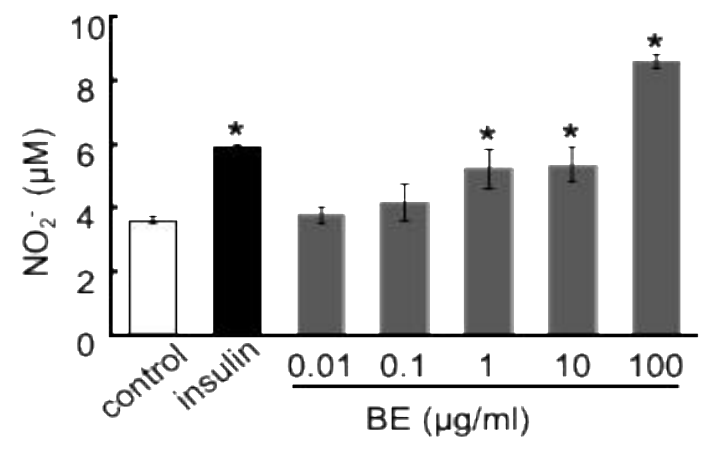

B

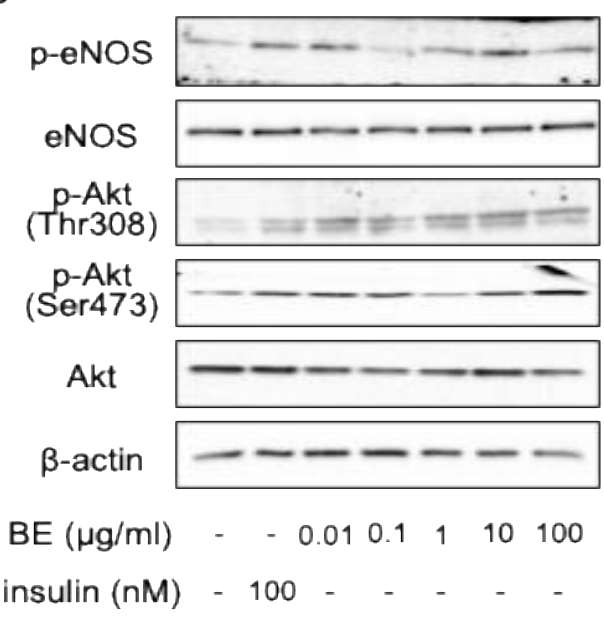

C
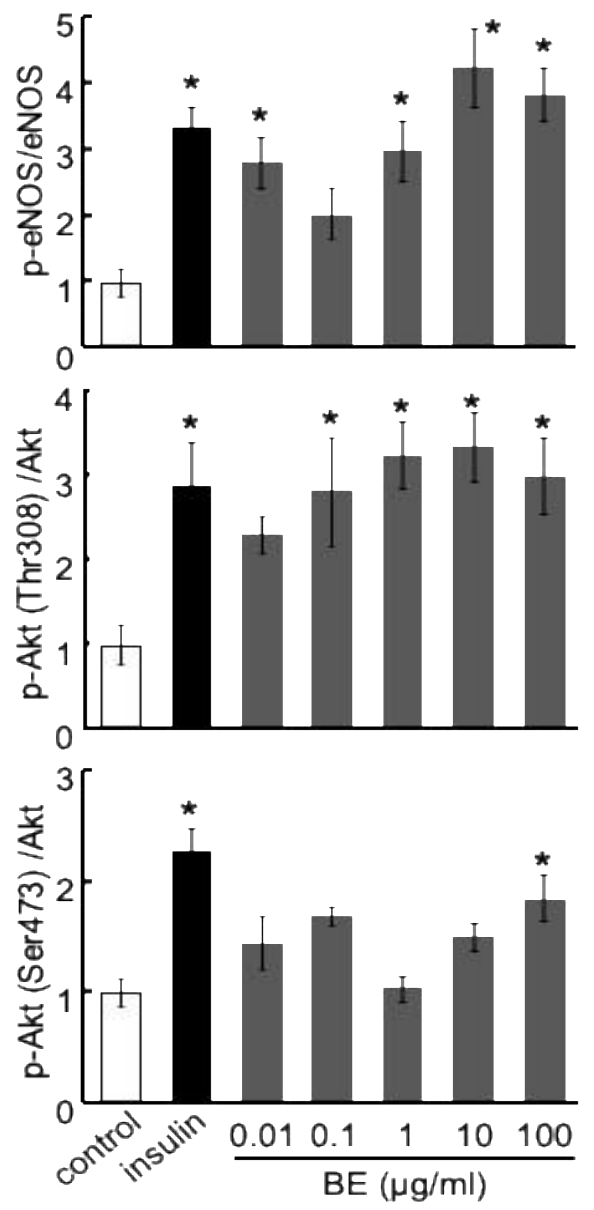

Figure 1. Effect of BE on NO production and its signaling pathways in HUVEC

HUVEC was treated with $0.01-100 \mu \mathrm{g} / \mathrm{ml} \mathrm{BE}$ or $100 \mathrm{nM}$ insulin as a positive control. A: NO concentrations in the medium were determined by the Griess method. Values are the mean $\pm S E(n=4)$. B and C; The phosphorylation level of eNOS and Akt were determined by western blot analysis. Each panel shows a typical result (B). The density of each phosphorylation and expression level was determined using Image J image analysis software (C). Values are the mean $\pm S E(n=3) .{ }^{*} p<0.05$ vs control (Dunnett's multiple comparison test). 
A
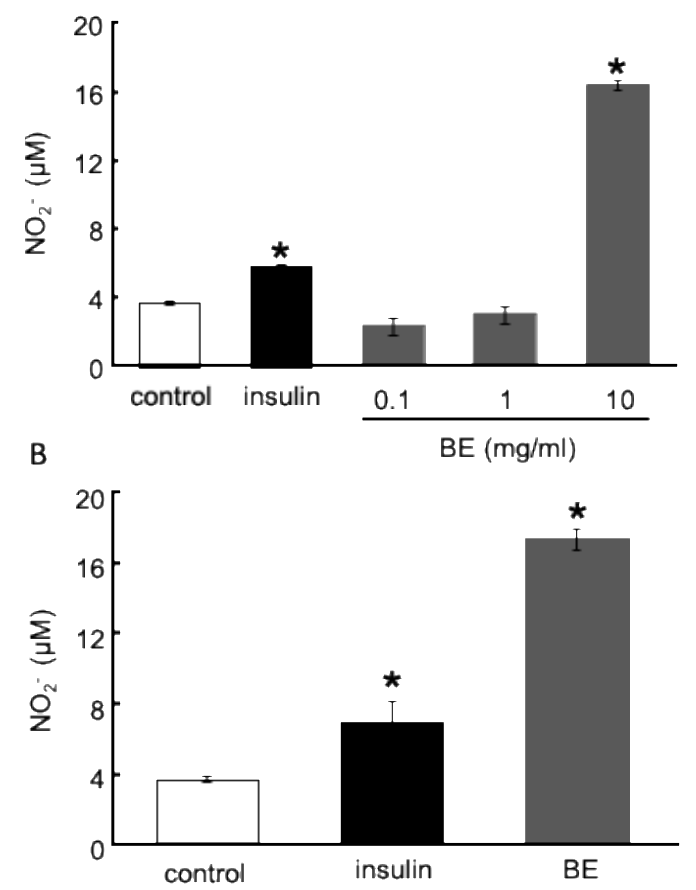

C

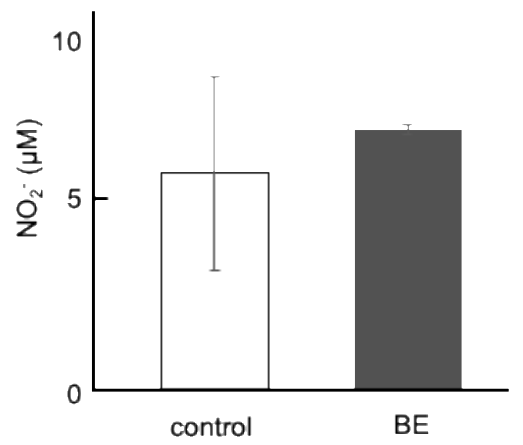

Figure 2. BE increased NO production in a co-culture system of Caco-2 cells and HUVEC.

A: Caco-2 cells were cultured on cell culture inserts and cocultured with HUVEC on the basolateral side of the transwell apparatus. BE was added to the apical side and incubated for $24 \mathrm{~h}$, and the cell culture medium from the basolateral side was collected to measure nitrite concentration.

B: HUVEC was directly treated with $300 \mu \mathrm{g} / \mathrm{ml} \mathrm{BE}$, which was almost equivalent to the concentration permeated through caco-2 cells or $100 \mathrm{nM}$ insulin as a positive control for $1 \mathrm{~h}$.

C: Caco-2 cells were cultured on cell culture inserts. BE was added to the apical side and incubated for $24 \mathrm{~h}$, and the cell culture medium from the basolateral side was collected. The polyphenols in permeated medium was removed and used to treat HUVEC for $1 \mathrm{~h}$. NO concentration in the medium was determined by Griess method. Values are the mean $\pm \mathrm{SE}(n=3)$, ${ }^{*} p<0.05$ vs control (Dunnett's multiple comparison test). medium from the basolateral side was measured.

As shown in Figure 2A, when $10 \mathrm{mg} / \mathrm{ml} \mathrm{BE}$ was added to Caco- 2 cells on the apical side of the apparatus, a significant increase in NO production was observed in the basolateral medium of HUVEC. This result suggested that flavan-3-ols permeated through the monolayer of Caco-2 cells, or certain substance(s) secreted from the Caco-2 cells may have contributed to the increase of NO production from HUVEC. When flavan-3-ols were removed from the basolateral medium by extraction with ethyl acetate, increased NO production from HUVEC was not observed (Figure 2C). This result suggested that flavan-3-ols that permeated through Caco-2 cells directly increased NO production from HUVEC. To confirm this phenomenon, HPLC was used to determine the flavan-3-ols that permeated through Caco- 2 cells after treating Caco- 2 cells on the apical side with BE at $10 \mathrm{mg} / \mathrm{ml}$ for $24 \mathrm{~h}$ (Table 1). About $3 \%$ of each flavan-3-ol was detected in their aglycone form under our experimental conditions, indicating that about $0.3 \mathrm{mg} / \mathrm{ml}$ of BE was estimated to be present in the basolateral medium of the co-culture system. It was confirmed that a significant increase in NO production was observed after direct treatment of HUVEC with 0.3 $\mathrm{mg} / \mathrm{ml}$ of $\mathrm{BE}$ to the same extent that $10 \mathrm{mg} / \mathrm{ml} \mathrm{BE}$ increased NO production in the co-culture system (Figure 2B).

Effects of flavan-3-ols in BE on NO production and eNOS phosphorylation in HUVEC: To specify the active compound in BE, HUVEC was treated with (-)epicatechin, procyanidin B2, procyanidin C1 or cinnamtannin $\mathrm{A} 2$ for $1 \mathrm{~h}$, after which NO production in 
Table 1. Concentration of flavan-3-ols after permeation through Caco-2 cells.

\begin{tabular}{|c|c|c|c|c|c|c|}
\hline \multirow[t]{2}{*}{ Flavan-3-ol (nM) } & \multicolumn{2}{|c|}{$\mathrm{BE} 0.1 \mathrm{mg} / \mathrm{ml}$} & \multicolumn{2}{|c|}{$\mathrm{BE} 1 \mathrm{mg} / \mathrm{ml}$} & \multicolumn{2}{|c|}{$\mathrm{BE} 10 \mathrm{mg} / \mathrm{ml}$} \\
\hline & aglycon & $\begin{array}{l}\text { aglycon + } \\
\text { conjugate }\end{array}$ & Aglycon & $\begin{array}{l}\text { aglycon + } \\
\text { conjugate }\end{array}$ & aglycon & $\begin{array}{l}\text { aglycon + } \\
\text { conjugate }\end{array}$ \\
\hline (-)-epicatechin & N.D. & $0.66 \pm 0.15$ & $2.37 \pm 0.52$ & $2.49 \pm 0.07$ & $67.6 \pm 6.3$ & $64.8 \pm 7.7$ \\
\hline procyanidin B2 & N.D. & N.D. & $0.58 \pm 0.11$ & $0.59 \pm 0.01$ & $24.0 \pm 2.4$ & $23.0 \pm 2.5$ \\
\hline procyanidin C1 & N.D. & N.D. & $0.18 \pm 0.02$ & $0.17 \pm 0.01$ & $6.67 \pm 0.74$ & $6.14 \pm 0.84$ \\
\hline cinnamtannin A2 & N.D. & N.D. & N.D. & N.D. & $2.84 \pm 0.50$ & $2.42 \pm 0.34$ \\
\hline
\end{tabular}

the cell culture medium was measured. Based on the previous in vivo results [16], the concentration of each flavan-3-ol in BE was set to the concentration that is possible to exist in the plasma of mice. As shown in Figure 3A, $0.5 \mu \mathrm{M}(-)$-epicatechin, $0.1 \mu \mathrm{M}$ procyanidin B2 and $0.05 \mu \mathrm{M}$ procyanidin C1 significantly increased NO production in the culture medium. On the other hand, $0.025 \mu \mathrm{M}$ cinnamtannin A2 did not increase NO production significantly. To investigate the upstream factors of NO production in HUVEC, the cells were treated with each flavan-3-ol for $30 \mathrm{~min}$ and western blotting analysis was performed. As shown in Figure 3B and C, $0.5 \mu \mathrm{M}(-)$-epicatechin, $0.1 \mu \mathrm{M}$ procyanidin B2 and $0.05 \mu \mathrm{M}$ procyanidin $\mathrm{C} 1$ significantly increased phosphorylation of eNOS in HUVEC. In addition, 0.1 $\mu \mathrm{M}$ procyanidin $\mathrm{B} 2$ and $0.05 \mu \mathrm{M}$ procyanidin $\mathrm{C} 1$ significantly increased phosphorylation of Akt at both of Thr308 and Ser473. Although a significant difference was not observed, $0.5 \mu \mathrm{M}(-)$-epicatechin also tended to increase phosphorylation of Akt at both Thr308 and Ser473. These results suggested that (-)-epicatechin, procyanidin B2 and procyanidin C1 had an ability to increase NO production via the Akt/eNOS pathway in HUVEC at low concentrations that are close to the plasma concentrations in mice after intake of BE [16].
To compare the effect of each flavan-3-ol in BE, HUVEC was treated with (-)-epicatechin, procyanidin $B 2$, procyanidin $\mathrm{C} 1$, or cinnamtannin $\mathrm{A} 2$ at the same concentration $(0.05 \mu \mathrm{M})$, and NO production and phosphorylation of Akt and eNOS were measured. As a result, procyanidin C1--not (-)-epicatechin, procyanidin B2 and cinnamtannin A2--significantly increased NO production in HUVEC via the Akt/eNOS pathway (Figure 4). Moreover, pretreatment with LY294002 ceased procyanidin C1-increased NO production (Figure 5). It is reported that $\mathrm{Ca}^{2+}$-dependent pathway is also involved in the NO production. Thus, HUVEC was treated with 0.05 or $50 \mu \mathrm{M}$ procyanidin $\mathrm{C} 1$ after preincubation with $5 \mu \mathrm{M}$ Fura2-AM, and intracellular $\mathrm{Ca}^{2+}$ level was measured using fluorescent microscopy. As shown in Figure 6, $0.05 \mu \mathrm{M}$ procyanidin $\mathrm{C} 1$ did not increase intracellular $\mathrm{Ca}^{2+}$ level, although $50 \mu \mathrm{M}$ procyanidin $\mathrm{C} 1$ increased it. Thus, procyanidin C1 increased mainly through the Akt/eNOS pathway, but not through $\mathrm{Ca}^{2+}$ dependent pathway, at a low concentration. From these results, it is suggested that procyanidin C1 was a main active compound in $\mathrm{BE}$ for promotion of the NO production via the Akt/eNOS pathway at the lower concentration that is close to the plasma concentration in mice after BE intake. 


\section{DISCUSSION}

Vascular dysfunction, which is caused by aging and vascular stiffness, is associated with a risk of developing CVD [1], and a reduction in NO levels can possibly trigger the onset of CVD. Black soybean seed coat contains an abundance flavan-3-ols, and these compounds have been reported to increase NO production in vascular endothelial cells [17-19]. However, these previous reports used flavan-3-ols at high concentrations. In this study, we demonstrated that BE directly increased NO production via the Akt/eNOS pathway in HUVEC at a low concentration (Figures 3 and 4). In addition, procyanidin C1 was the most effective compound in BE at increasing NO production at the lower concentration (Figure 4). To the best of our knowledge, this is the first report demonstrating flavan-3-ols, in particular procyanidin C1, increasing NO production in HUVEC at a concentration that is close to the plasma concentration in mice after intake of flavan-3-ols-rich food materials.

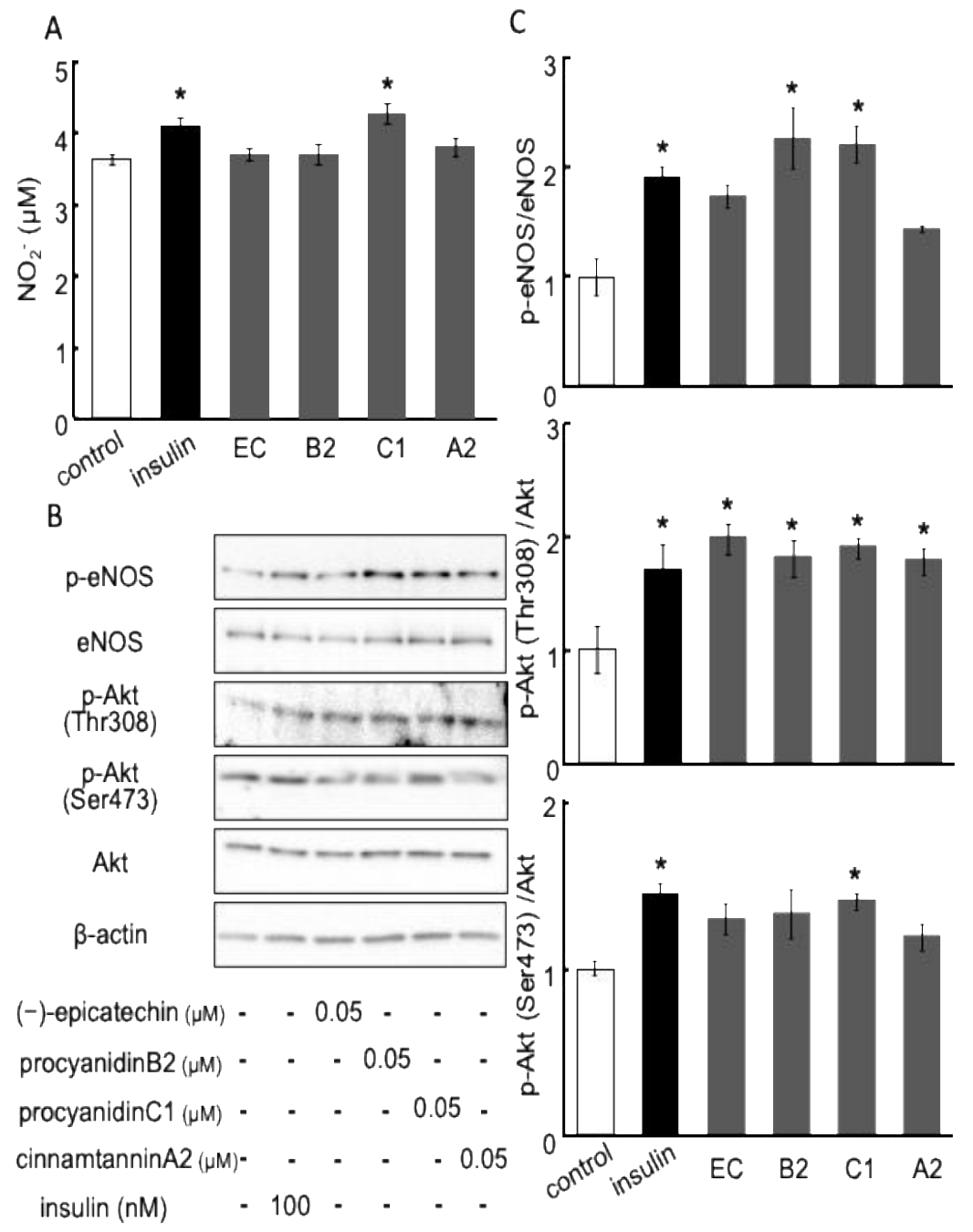

Figure 3. Flavan-3-ols contained in BE increased NO production through Akt/eNOS signaling pathway.

HUVEC was treated with $0.5 \mu \mathrm{M}$ (-)-epicatechin, $0.1 \mu \mathrm{M}$ procyanidinB2, $0.05 \mu \mathrm{M}$ procyanidinC1 and $0.025 \mu \mathrm{M}$ cynnamtannin $\mathrm{A} 2$ or $100 \mathrm{nM}$ insulin as a positive control. A; NO concentration in the medium was determined by Griess method. B and C; The phosphorylation level of eNOS and Akt were determined by western blot analysis. Each panel shows a typical result (B). The density of each phosphorylation and expression level was determined using Image J image analysis software (C). Values are the mean $\pm S E(n=3),{ }^{*} p<0.05$ vs control (Dunnett's multiple comparison test). 


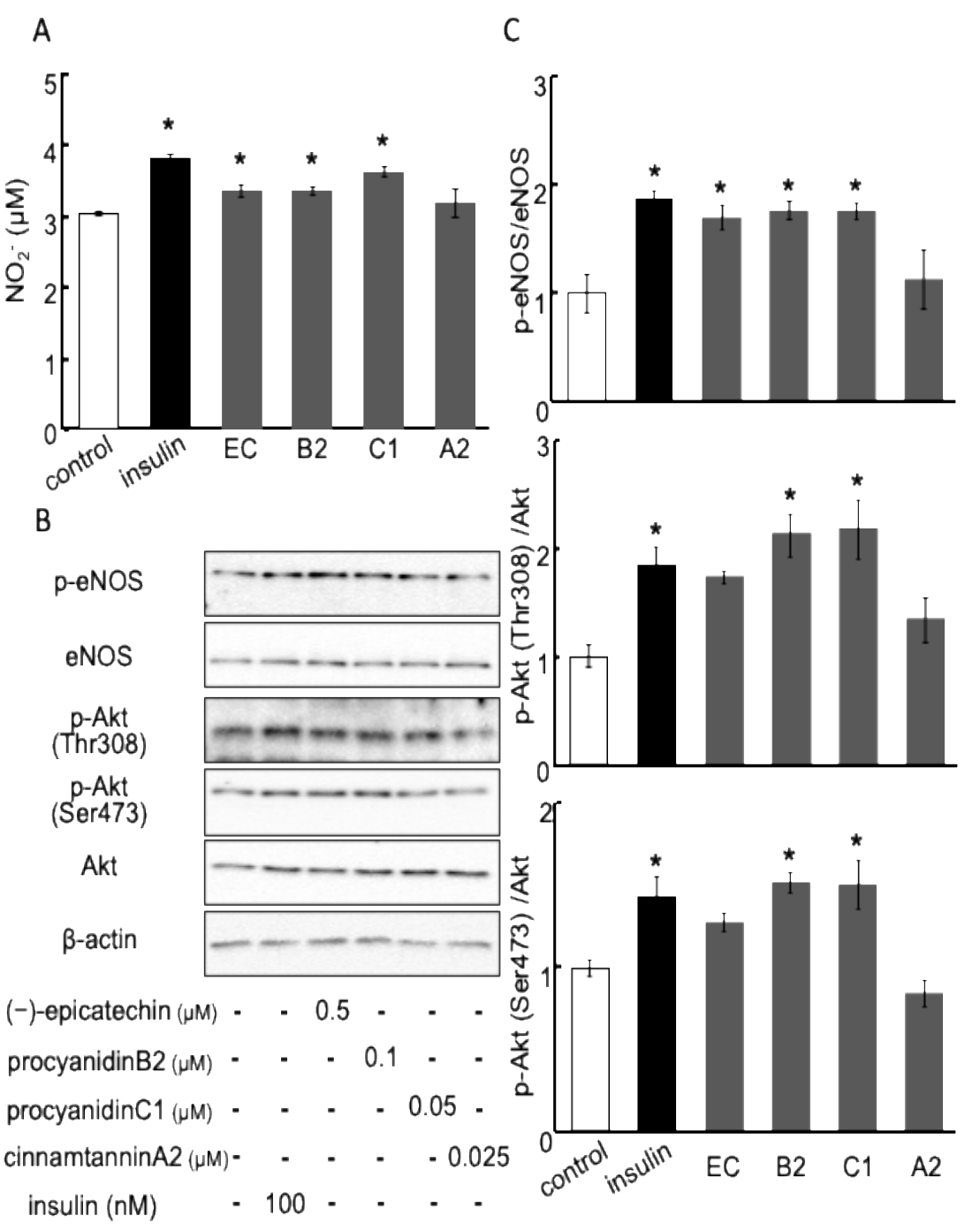

Figure 4. Procyanidin C1 increased NO production thorough the Akt/eNOS signaling pathway.

HUVEC was treated with (-)-epicatechin, procyanidinB2, procyanidinC1 and cynnamtannin $\mathrm{A} 2$ at $0.05 \mu \mathrm{M}$ or $100 \mathrm{nM}$ insulin as a positive control. A: NO concentration in the medium was determined by Griess method. The phosphorylation level of eNOS and Akt were determined by western blot analysis. B and C; Each panel shows a typical result (B). The density of each phosphorylation and expression level was determined using Image $J$ image analysis software (C). Values are the mean \pm SE $(n=3), * p<0.05$ vs control (Dunnett's multiple comparison test).

In this study, about $3 \%$ of flavan-3-ols in BE permeated through the intestinal epithelial cells (Table 1) and promoted the NO production in HUVEC (Figure 2). The passive diffusion across the intestinal epithelium can occur either through the transcellular route or the paracellular route [24]. It has been reported that highly hydrophilic compounds prefer the paracellular route and enter the body through tight junctions [24]. Since flavan-3-ols containing in BE are hydrophilic, they would be absorbed through the paracellular route. A previous study reported that flavan-3-ols in cocoa powder extract affect the function of tight junctions and permeated through intestinal epithelial cells by the paracellular route [25]. In the present study, the sum of flavan-3-ols that had permeated reached about $100 \mathrm{nM}$ after treatment with $10 \mathrm{mg} / \mathrm{ml}$ of BE to Caco-2 cells. Recently, we have reported that about $100 \mathrm{nM}$ flavan-3-ols are detected 


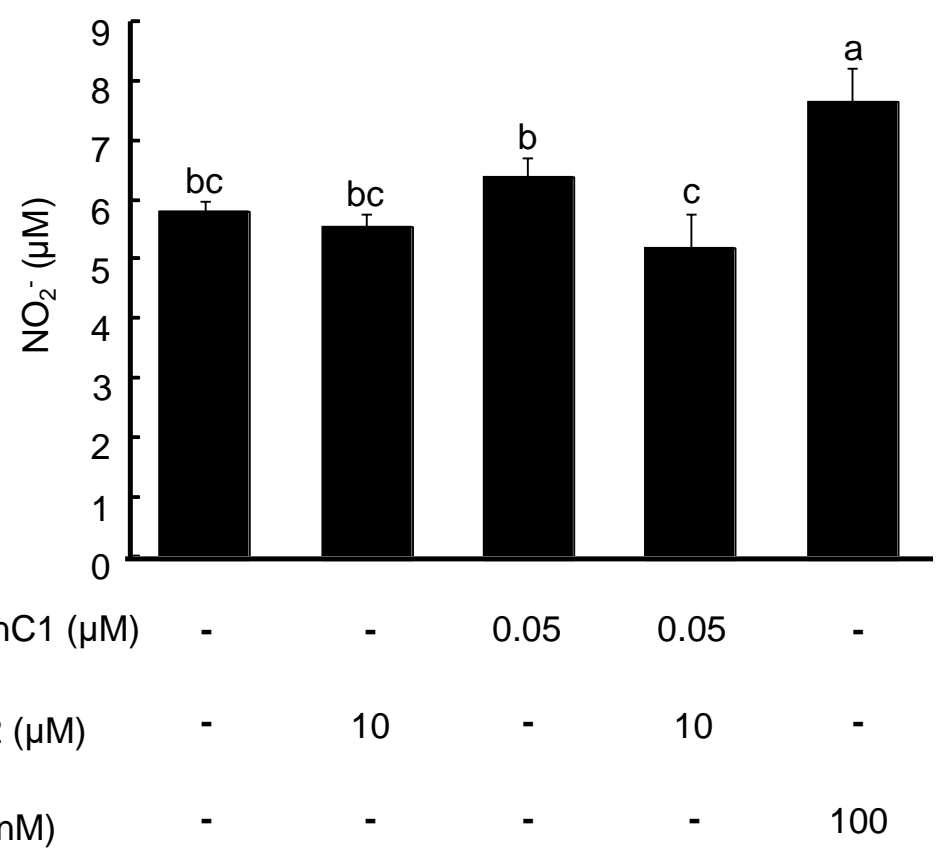

Figure 5. Procyanidin C1-induceed NO production was canceled by a PI3K inhibitor.

HUVEC was pretreated with or without $10 \mu \mathrm{M}$ PI3K inhibitor (LY294002) for $1 \mathrm{~h}$ and then $0.05 \mu \mathrm{M}$ procyanidinC1 or $100 \mathrm{nM}$ insulin as a positive control were treated to the cells. NO concentration in the medium was determined by Griess method. Values are the mean $\pm S E(n=3)$. Means with different letters differ significantly $(p<0.05 ;$ Tukey's HSD test).

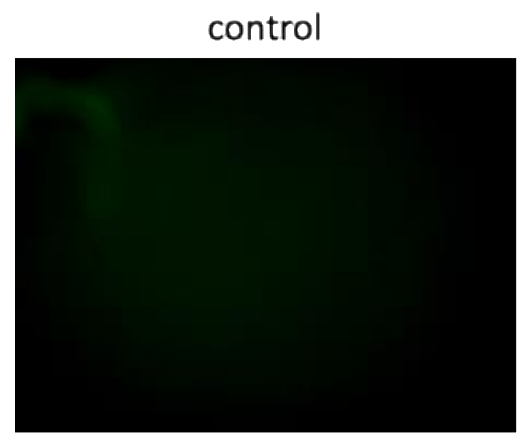

Procyanidin $\mathrm{C} 10.05 \mu \mathrm{M}$

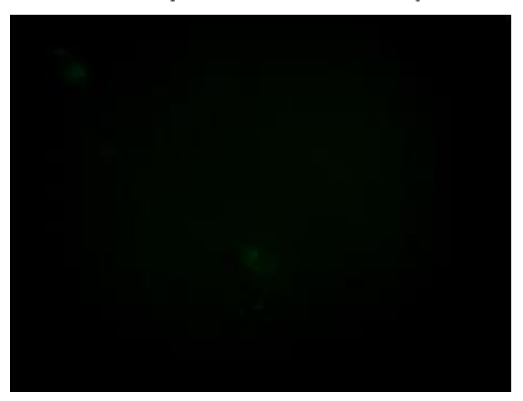

A23187

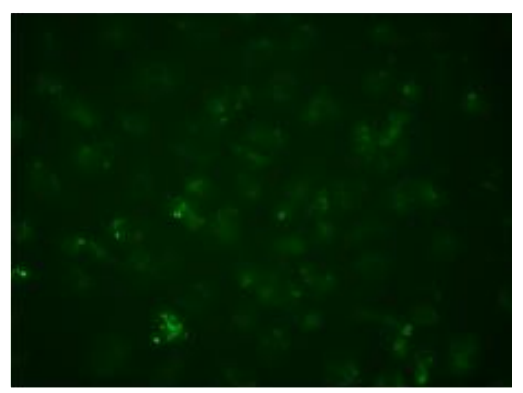

Procyanidin C1 50 $\mu \mathrm{M}$

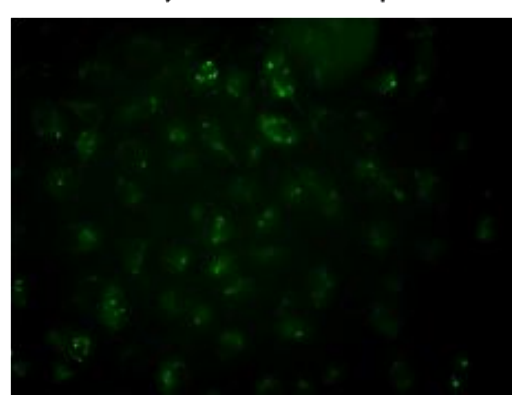

Figure 6. The effect of procyanidin $\mathrm{C} 1$ on intracellular $\mathrm{Ca}^{2+}$ levels.

HUVEC was incubated with 0.05 or $50 \mu \mathrm{M}$ procyanidin C1 or A23187 (Calcium lonophore) as a positive control for 10 min, after preincubation with Fura2-AM. The intracellular $\mathrm{Ca}^{2+}$ level was then estimated by a fluorescence microscope. 
in the human plasma after the intake of $30 \mathrm{~g} /$ day black soybean for 8 weeks [14]. Moreover, almost the same concentration of flavan-3-ols was detected as the aglycone form after orally administered $250 \mathrm{mg} / \mathrm{kg} \mathrm{B.W}$. of $B E$ in ICR mice. Therefore, the concentration of BE used in the present study was relevant to detected concentrations of BE-derived flavan-3-ols in both human and animal experiments.

Among the flavan-3-ols that had permeated in the co-culture system, procyanidin C1 was the most effective compound in BE for NO production through the Akt/eNOS pathway, but not through Ca2+dependent pathway, and a minimum effective concentration of it was $0.05 \mu \mathrm{M}$ (Figure 6). Similarly, a previous study reported that $50 \mu \mathrm{M}$ procyanidin $\mathrm{C} 1$ activated eNOS mainly through Ca2+-dependent pathway, and its effect was stronger than (-)epicatechin and procyanidin B2 [17]. However, such a high concentration of procyanidin $\mathrm{C} 1$ is difficult to find in the body after oral intake of flavan-3-ol-rich food materials or procyanidin C1 itself. From our results and the results from the previous study mentioned, we can, at least, safely speculate that procyanidin $\mathrm{C} 1$ is the most effective compound among flavan-3-ols. It is, however, unclear what procyanidin C1-specific target molecule leads to the activation of signaling pathway(s) for NO production. We assume that the core structure of procyanidins (polymerized 2-phenyl-3,4-dihydro-2Hchromen-3-ol) interacts with a target molecule for Akt, such as insulin-like growth factor-1 receptor (IGF-1R) [26] or epidermal growth factor receptor (EGFR) [27]. A previous study reported that procyanidins from Cinnamomi Cortex activated IGF-1R in vitro [28]. Another study reported that oligomer procyanidins from grape seed activated EGFR in vitro and in vivo [29]. Further study is needed to search for the target molecule of procyanidin C1 and its underlying molecular mechanism in future.

In this study, HUVEC was treated with flavan-3-ols in $\mathrm{BE}$ at a concentration that can exist as the aglycon form in plasma [16] because we only detected the aglycon form of them in the basolateral medium of the co-culture system. However, it has been reported that flavan-3-ols in BE exist mainly as conjugate forms in the plasma [16]. Thus, future study should clarify whether the conjugated forms of flavan-3-ols increase the NO production in vascular endothelial cells.

\section{CONCLUSION}

In conclusion, a portion of flavan-3-ols in BE directly promotes the NO production from vascular endothelial cells through the Akt/eNOS pathway at a low concentration that close to the plasma concentration in mice after intake of $\mathrm{BE}$. In addition, procyanidin $\mathrm{C} 1$ is the most effective compound among the flavan-3-ols in BE in promoting NO production. Our findings indicate the possibility that flavan-3-ols in the black soybean seed coat may prevent CVD by improving vascular function.

List of abbreviations used: NO; nitric oxide, eNOS; endothelial nitric oxide synthase, BE; black soybean seed coat extract, CVD; cardiovascular diseases, Akt; phosphoinositide 3-kinase, PI3K; phosphoinositide 3kinase, PBS; phosphate-buffered saline

Competing interests: There are no competing interests in this study.

Authors' Contributions: Yamashita Y. designed the research. Chiaki Domae, Yamashita $Y$ conducted the experiments and statistical analysis. Chiaki Domae, Yamashita $\mathrm{Y}$, and Ashida $\mathrm{H}$. wrote the manuscript. All 
authors have read and approved the final version of the manuscript.

Acknowledgments and Funding: This work was supported by the Cross-ministerial Strategic Innovation Promotion Program by Cabinet Office, Government of Japan, and JSPS KAKENHI Grant-in-Aid under Grant Numbers 17K15269 (Y.Y.) and 17H00818 (H.A.).

\section{REFERENCES}

1. Moore RJ, Jackson KG, Minihane AM. Green tea (Camellia sinensis) catechins and vascular function. Br J Nutr. 2009, 102(12):1790-1802.

2. Ding $H$, Hashem $M$, Triggle $C$. Increased oxidative stress in the streptozotocin-induced diabetic apoE-deficient mouse: changes in expression of NADPH oxidase subunits and eNOS. Eur J Pharmacol. 2007, 561(1-3):121-128.

3. Tabit CE, Chung WB, Hamburg NM, Vita JA. Endothelial dysfunction in diabetes mellitus: Molecular mechanisms and clinical implications. Rev Endocr Metab Disord. 2010, $11(1): 61-74$

4. Bakker W, Eringa EC, Sipkema P, van Hinsbergh VW. Endothelial dysfunction and diabetes: roles of hyperglycemia, impaired insulin signaling and obesity. Cell Tissue Res. 2009, 335(1):165-189.

5. Michel T, Vanhoutte PM. Cellular signaling and NO production. Pflugers Arch. 2010, 459(6):807-816.

6. Ghimire K, Altmann HM, Straub AC, Isenberg JS. Nitric oxide: what's new to NO? Am J Physiol Cell Physiol. 2017 312(3):C254-C262

7. Wilmes V, Scheiper S, Roehr W, Niess C, Kippenberger S, Steinhorst K, Verhoff MA, Kauferstein S. Increased inducible nitric oxide synthase (iNOS) expression in human myocardial infarction. Int J Legal Med. 2020, 134(2):575581.

8. Cai H, Liu D, Garcia JG. CaM Kinase II-dependent pathophysiological signalling in endothelial cells. Cardiovasc Res. 2008, 77(1):30-34

9. Fulton D, Gratton JP, McCabe TJ, Fontana J, Fujio Y, Walsh K, Franke TF, Papapetropoulos A, Sessa WC. Regulation of endothelium-derived nitric oxide production by the protein kinase Akt. Nature. 1999, 399(6736):597-601.

10. Todd JJ, Vodkin LO. Pigmented Soybean (Glycine max) Seed Coats Accumulate Proanthocyanidins during Development. Plant Physiol. 1993, 102(2):663-670.

11. Choung MG, Baek IY, Kang ST, Han WY, Shin DC, Moon HP, Kang KH. Isolation and Determination of Anthocyanins in Seed Coats of Black Soybean (Glycine max (L.) Merr.). J Agric Food Chem. 2001, 49(12):5848-5851.

12. Astadi IR, Astuti M, Santoso U, Nugraheni PS. In vitro antioxidant activity of anthocyanins of black soybean seed coat in human low density lipoprotein (LDL). Food Chem. 2009, 112(3):659-663.

13. Kanamoto $\mathrm{Y}$, Yamashita $\mathrm{Y}$, Nanba F, Yoshida T, Tsuda T, Fukuda I, Nakamura-Tsuruta S, Ashida H. A black soybean seed coat extract prevents obesity and glucose intolerance by up-regulating uncoupling proteins and down-regulating inflammatory cytokines in high-fat diet-fed mice. J Agric Food Chem. 2011, 59(16):8985-8993.

14. Yamashita Y, Wang L, Nakamura A, Nanba F, Saito S, Toda T, Nakagawa J, Ashida H. Black Soybean Improves the Vascular Function Through an Increase in Nitric Oxide and a Decrease in Oxidative Stress in Healthy Women. Arch Biochem Biophys. 2020, in press.

15. Domae C, Nanba F, Maruo T, Suzuki T, Ashida H, Yamashita Y . Black soybean seed coat polyphenols promote nitric oxide production in the aorta through glucagon-like peptide-1 secretion from the intestinal cells. Food Funct. 2019, 10 (12), 7875-7882

16. Wang L, Yamashita Y, Komeda S, Saito A, Ashida H. Absorption, metabolism, distribution and faecal excretion of B-type procyanidin oligomers in mice after a single oral administration of black soybean seed coat extract. Food Funct. 2018, 9(10):5362-5370.

17. Byun EB, Ishikawa T, Suyama A, Kono M, Nakashima S, Kanda T, Miyamoto T, Matsui T. A procyanidin trimer, C1, promotes NO production in rat aortic endothelial cells via both hyperpolarization and PI3K/Akt pathways. Eur J Pharmacol. 2012, 692(1-3):52-60

18. Feng Z, Wei RB, Hong Q, Cui SY, Chen XM. Grape seed extract enhances eNOS expression and NO production through regulating calcium-mediated AKT phosphorylation in $\mathrm{H}_{2} \mathrm{O}_{2}$-treated endothelium. Cell Biol Int. 2010, 
34(10):1055-1061.

19. Kaufeld AM, Pertz HH, Kolodziej H. A chemically defined 2,3-trans procyanidin fraction from willow bark causes redox-sensitive endothelium-dependent relaxation in porcine coronary arteries. J Nat Prod. 2014, 77(7):16071614.

20. Ito C, Oki T, Yoshida T, Nanba F, Yamada K, Toda T. Characterisation of proanthocyanidins from black soybeans: isolation and characterisation of proanthocyanidin oligomers from black soybean seed coats. Food Chem. 2013, 141(3):2507-2512.

21. Swain T, Hillis WE. The phenolic constituents of Prunus domestica. I. The quantitative analysis of phenolic constituents. J Sci Food Agric. 1959, 10(1):63-68.

22. Takahashi A, Yamamoto N, Murakami A. Cardamonin suppresses nitric oxide production via blocking the IFNY/STAT pathway in endotoxin-challenged peritoneal macrophages of ICFR mice. Life Sci. 2011, 89(9-10):337-342.

23. Wang L, Yamashita Y, Saito A, Ashida H. An analysis method for flavan-3-ols using high performance liquid chromatography coupled with a fluorescence detector. J Food Drug Anal. 2017, 25(3):478-487

24. Linnankoski J, Mäkelä J, Palmgren J, Mauriala T, Vedin C, Ungell AL, Lazorova L, Artursson P, Urtti A, Yliperttula M. Paracellular porosity and pore size of the human intestinal epithelium in tissue and cell culture models. J Pharm Sci.
2010, 99(4):2166-2675.

25. Kosińska A, Andlauer W. Cocoa polyphenols are absorbed in Caco-2 cell model of intestinal epithelium. Food Chem. 2012, 135(3):999-1005.

26. Nakagawa Y, Ishimura K, Oya S, Kamino M, Fujii Y, Nanba F, Toda T, Ishii T, Adachi T, Suhara Y, Osakabe N. Comparison of the sympathetic stimulatory abilities of B-type procyanidins based on induction of uncoupling protein-1 in brown adipose tissue (BAT) and increased plasma catecholamine (CA) in mice. Plos One. 2018, 13(7):e0201203.

27. Fujii Y, Suhara Y, Sukikara Y, Teshima T, Hirota Y, Yoshimura $\mathrm{K}$, Osakabe N. Elucidation of the Interaction between Flavan-3-ols and Bovine Serum Albumin and Its Effect on Their In-Vitro Cytotoxicity. Molecules. 2019, 24(20):3667.

28. Ohnuma T, Sakamoto K, Shinoda A, Takagi C, Ohno S, Nishiyama T, Ogura K, Hiratsuka A. Procyanidins from Cinnamomi Cortex promote proteasome-independent degradation of nuclear Nrf2 through phosphorylation of insulin-like growth factor-1 receptor in A549 cells. Arch Biochem Biophys. 2017, 635:66-73.

29. Zheng HL, Wang LH, Sun BS, Li Y, Yang JY, Wu CF. Oligomer procyanidins (F2) repress HIF-1 $\alpha$ expression in human U87 glioma cells by inhibiting the EGFR/AKT/mTOR and MAPK/ERK1/2 signaling pathways in vitro and in vivo. Oncotarget. 2017, 8(49):85252-85262. 